Address for Correspondence:

Dr. Ayoub Innabi, MD. Department of

Internal Medicine. University of Arkansas

for Medical Sciences, Arkansas.

4301 W. Markham Mail Slot \#634,

Little Rock, AR 72205, USA.

Email: ayoubinnabi@yahoo.com

\begin{tabular}{|l|}
\hline Access this article online \\
\hline Website: \\
www.intern-med.com \\
\hline DOI: \\
10.1515/jtim-2017-0014 \\
\hline Quick Response Code: \\
\hline \\
\\
\end{tabular}

\title{
Prediction of survival following percutaneous biliary drainage for malignant biliary obstruction
}

\author{
Wa'el Tuqan ${ }^{1}$, Ayoub Innabi ${ }^{2}$, Alia Alawneh ${ }^{3}$, Fadi Abu Farsakh ${ }^{4}$, Maan Al-Khatib ${ }^{5}$ \\ 'Department of Internal Medicine, University of New Mexico School of medicine, \\ Albequerque, New Mexico, USA; \\ ${ }^{2}$ Department of Internal Medicine, University of Arkansas for Medical Sciences, Arkansas, USA; \\ ${ }^{3}$ Department of Medical Oncology, Tawam Hospital, Abu Dhabi, United Arab Emirates; \\ ${ }^{4}$ Department of Medical Oncology, King Hussein Cancer Center, Amman, Jordan; \\ ${ }^{5}$ Department of Radiology, Tawam Hospital, Abu Dhabi, United Arab Emirates
}

\begin{abstract}
Background and Objectives: Percutaneous transhepatic biliary drain is an intervention used to relieve malignant biliary obstruction. This study aims to explore survival after biliary drain insertion, predictive factors of survival and effectiveness to reduce total bilirubin level. Methods: We conducted a retrospective analysis of 72 patients who had malignant biliary obstruction and received biliary drain during the time period between March 2005 and February 2015. Results: Median patients' age was 56 years. 38 (52.7\%) were males, 34(47.2\%) were females. Median survival post biliary drain insertion was 46 days, $95 \% \mathrm{C} / \mathrm{l}$ (37.92-54.02), range (2-453 days). 1 , 3 , and 6 month survival rates were $64.7 \%, 26.5 \%$, and $7.4 \%$ respectively. Multivariate analysis by Cox proportional hazards regression model showed the presence of ascites to be significant predictors of survival, other factors analyzed were: total bilirubin, serum creatinine, international normalization ratio, serum albumin, pleural effusion and liver metastasis. Conclusion: Survival after biliary drain insertion can vary from few days to few months. Presence of ascites is an independent predictor of survival after this intervention.
\end{abstract}

Key words: biliary, drain, malignant, survival, prognosis

\section{INTRODUCTION}

Percutaneous transhepatic cholangiography is a procedure that was developed from a diagnostic procedure to an interventional procedure where the biliary tract can be drained. ${ }^{[1-7]}$ Percutaneous transhepatic biliary drainage (PTBD) can be used in benign and malignant biliary obstruction (MBO). It was shown to be effective, and many times, it was the rescue procedure for inoperable patients, in whom the endoscopic procedure cannot be performed due to poor general state, anatomical conditions or access failure..$^{[8]}$ Most of the times, malignant tumors causing bile duct obstruction have a very poor prognosis. ${ }^{[9,10]}$ The primary tumors associated with MBO include biliary tract, pancreatic, hepatocellular and duodenal. Metastasis to the porta hepatis from gastric, colon, and breast cancer is another cause of MBO. ${ }^{[11,12]}$

PTBD was shown to be effective in reducing the serum bilirubin level to a degree that patients could receive additional chemotherapy. ${ }^{[13]}$ In other patients where chemotherapy cannot be offered, PTBD may have a palliative value in relieving biliary obstruction. PTBD was shown in a prospective study done by Robson et al. to significantly reduce the severity of pruritus. ${ }^{[14]}$ However, there is mixed results with regards to the effect of PTBD on the quality of life. PTBD is an intervention that is not free of complications. Most common complication following the biliary drainage is drain dislodgement and the need for re-intervention. Other possible complications include pain, biliary leakage 
around puncture site, hemorrhage, perforation, infections and stent migration. ${ }^{[8,9,15,16]}$ Therefore, it is really important to weigh the potential benefits and risks of this procedure in patients with $\mathrm{MBO}$.

Short term survival was reported in patients who underwent PTBD for malignant biliary obstruction. ${ }^{[15,17,18]}$ Factors able to predict the long and short term survival after PTBD are scarcely reported in the medical literature and have not yet been well established. ${ }^{[13]}$ In this study, we aimed to find prognostic factors of short term survival after PTBD in cancer patients; in addition, we aimed to evaluate the effectiveness of this procedure during the last month of life.

\section{METHODS}

This is a retrospective cohort study. All patients who had malignant biliary obstruction and received biliary drain during the time period between March 2005 and February 2015 were eligible for inclusion. Patients were identified by using the interventional radiology quality data, this data was collected prospectively. Patient characteristics and laboratory data were obtained retrospectively using the electronic medical database.

The following clinical and laboratory data were collected: age at the time of drainage, gender, diagnosis, cause of obstruction (primary disease versus metastatic disease), presence of liver metastasis, pleural effusion, ascites, serum albumin level, internal normalization ratio (INR), serum creatinine level and total serum bilirubin level before drainage. In addition, we collected total serum bilirubin one month after PTBD.

According to current literature, ${ }^{[11,19,20]}$ we tested the following factors for prognostic value of short term survival following PTBD: international standardized ratio, serum albumin at the time of insertion, serum creatinine at the time of insertion, serum total bilirubin level, presence of ascites, presence of plural effusion, and liver metastasis.

We compared the median total serum bilirubin level before and after PTBD. We classified the patients according to their survival into two groups: patients who survived less than 30 days (group 1) and patients who survived more than 30 days (group 2). We compared the difference in total serum bilirubin level before and after PTBD in both groups.

This study was carried in compliance with the Declaration of Helsinki and was approved by the Institutional Review Board at King Hussein Cancer Center.

\section{Statistical analysis}

Kaplan Meier curves were used to draw survival curves and assess survival rates. Log rank test was used to analyze the relationship between categorical variables and survival times. Statistically significant predictors $(P<0.05)$ and continuous variables were entered into a multivariate analysis using a Cox proportional hazards regression model; backward elimination technique was used. Wilcoxon signed-rank test and Mann-Whitney test were used to compare the median total serum bilirubin levels. IBM SPSS version 21 was used.

\section{RESULTS}

Our records showed that 72 patients with malignant biliary obstruction received biliary drain between March 2005 and February 2015.

Median patients' age was 56 years. 38 (52.7\%) were males. In most cases, the cause of obstruction was due to metastasis to the liver (32.8\%). Primary cancers that caused biliary obstruction were pancreatic $(24.6 \%)$, gastric $(17.8 \%)$, cancer of unknown origin $(10 \%)$, colon $(7 \%)$, breast $(7 \%)$, Lymphoma (4\%), gallbladder (4\%), small intestine (4\%), and others including uterine, renal, ovarian, rectal and lung. Table 1 shows the patients' characteristics.

In this cohort, the survival rates at one month, 3 months, 6 months and 12 months were $64 \%, 27 \%, 7 \%$, and $1 \%$, respectively, with median survival of 46 days; $95 \%$ confidence interval (37.92-54.08), Figure 1. Using the multivariate cox proportional hazard model, the presence of ascites and international standardized ratio showed statistically significant association with survival following PTBD. The following factors did not show statistically significant association with survival: serum albumin level at the time of insertion, serum creatinine level at the time of insertion, serum total bilirubin level, presence of pleural effusion, and liver metastasis (Table 2).

Median total serum bilirubin level before the intervention was $15.01 \mathrm{mg} / \mathrm{dL}$, median total serum bilirubin level after the intervention was $6.4 \mathrm{mg} / \mathrm{dL}, P$ value $<0.01$ by Wilcoxon signed ranks test. Patients who survived less than 30 days (group 1) had statistically significant different total bilirubin levels before and after the intervention, $17.5 \mathrm{mg} / \mathrm{dL}$ and $6.8 \mathrm{mg} / \mathrm{dL}$ respectively, and $P$ value < 0.05. Patients who survived more than 30 days (group 2) had statistically significant different total bilirubin level before and after the intervention, $14.5 \mathrm{mg} / \mathrm{dL}$ and $5.8 \mathrm{mg} / \mathrm{dL}$ respectively, and $P$ value $<0.05$. Mann-Whitney test was used to compare group 1 and group 2 in terms of difference in serum bilirubin level after biliary drainage; no statistically significant difference was found. 
Tuqan et al.: Biliary drainage in malignant obstruction

\begin{tabular}{|c|c|c|c|c|c|c|c|}
\hline & \multirow[t]{2}{*}{ Mean } & \multirow[t]{2}{*}{ Median } & \multirow[t]{2}{*}{ Minimum } & \multirow[t]{2}{*}{ Maximum } & \multicolumn{3}{|c|}{ Percentiles } \\
\hline & & & & & 25 & 50 & 75 \\
\hline Age (years) & 57 & 56 & 30 & 84 & 49 & 56 & 64 \\
\hline INR & 1.25 & 1.22 & 0.94 & 1.92 & 1.08 & 1.22 & 1.40 \\
\hline Albumin (g/dL) & 2.72 & 2.70 & 1.30 & 3.80 & 2.40 & 2.70 & 3.10 \\
\hline $\operatorname{ALT}(\mathrm{U} / \mathrm{L})$ & 104.24 & 85.50 & 21.00 & 454.00 & 49.25 & 85.50 & 129.00 \\
\hline ALP (U/L) & 744.58 & 569.00 & 48.00 & 6115.00 & 366.00 & 569.00 & 779.50 \\
\hline Urea $(\mathrm{mg} / \mathrm{dL})$ & 37.97 & 26.00 & 5.00 & 173.00 & 18.25 & 26.00 & 42.50 \\
\hline Creatinine (mg/dL) & 0.97 & 0.80 & 0.30 & 4.60 & 0.60 & 0.80 & 0.90 \\
\hline Platelets $\times 10^{3} / \mathrm{mL}$ & 287.00 & 258.50 & 29.00 & 696.00 & 205.50 & 258.50 & 345.75 \\
\hline Total serum bilirubin (mg/dL) & 16.20 & 15.01 & 0.90 & 46.40 & 9.02 & 15.01 & 20.93 \\
\hline
\end{tabular}

INR: international normalization ratio; ALT: alanine aminotransferase; ALP: alkaline phosphatase.

\begin{tabular}{|c|c|c|c|c|}
\hline \multirow{2}{*}{$\frac{\text { Factor }}{\text { INR }}$} & \multirow{2}{*}{$\begin{array}{l}\text { Hazard ratio } \\
0.281\end{array}$} & \multicolumn{2}{|c|}{ Confidence interval } & \multirow{2}{*}{$\begin{array}{l}\boldsymbol{P} \text { value } \\
0.029\end{array}$} \\
\hline & & 0.090 & 0.876 & \\
\hline Serum albumin level & 1.081 & 0.665 & 1.758 & 0.754 \\
\hline Serum creatinine level & 1.347 & 0.993 & 1.826 & 0.056 \\
\hline Presence of ascites & 0.462 & 0.260 & 0.820 & 0.008 \\
\hline Presence of pleural effusion & 1.519 & 0.811 & 2.844 & 0.192 \\
\hline Liver metastasis & 0.770 & 0.437 & 1.355 & 0.365 \\
\hline Serum total bilirubin level & 1.016 & 0.983 & 1.050 & 0.345 \\
\hline
\end{tabular}

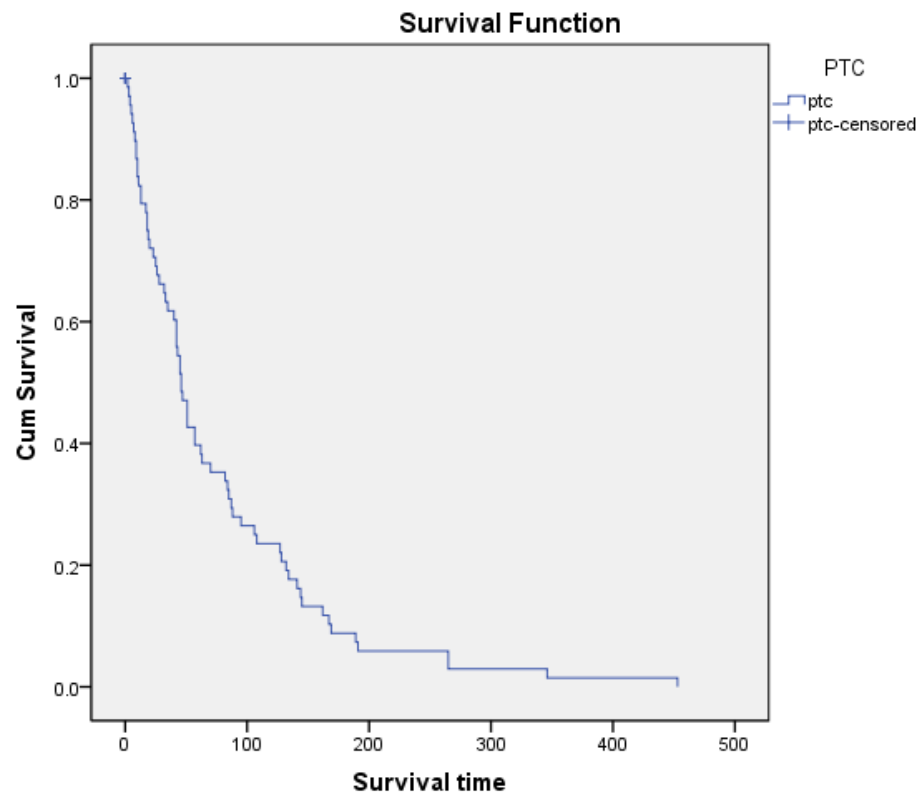

Figure 1: Survival rates at one month, 3 months, 6 months and 12 months were $64 \%, 27 \%$, 7\%, and $1 \%$, respectively with median survival of 46 days; $95 \%$ Confidence Interval (37.92-54.08)

\section{DISCUSSION}

Malignant obstructive jaundice in majority of patients is incurable and indicates a poor prognosis. ${ }^{[10,21]}$ Percutaneous biliary drainage is a method for palliative treatment considered in these patients, which relieves the high serum bilirubin related symptoms and provides them with an opportunity to receive additional therapies. However, PTBD is an invasive procedure and has its own risks and complications. ${ }^{[2]}$ Previous studies have described that obstructive jaundice with poor prognostic factors could not receive survival benefit from PTBD, even though they achieved an improvement of their liver dysfunction. ${ }^{[23]}$ For this reason, few studies investigated the 
prognostic factors that predict short survival time in these patients. ${ }^{[11,13,19,23]}$ By identifying poor predictive survival factors, we believe that through pre-PTBD clinical, radiological, and laboratory data analysis, we can identify the population of patients for whom PTBD will not increase well-being or survival, and in return, such invasive procedure can be avoided. In this study, we aimed to address two main issues: the survival of patients with malignant biliary obstruction post percutaneous drainage; and to describe the prognostic factors that predict short survival time after percutaneous biliary drainage.

In this cohort, survival rates at 1 month, 3 months, 6 months and 12 months were $64 \%, 27 \%, 7 \%$, and $1 \%$, respectively, with overall median survival of 46 days. Our median survival time is low compared to other studies, which can be explained by poor general condition of our patients and low rate of patients who received chemotherapy. ${ }^{[1]}$ Only 10 patients in our cohort were eligible to receive subsequent chemotherapy post procedure. Other studies reported increase survival time after chemotherapy administration post percutaneous biliary drainage. ${ }^{[13]}$ For example, Kasuga et al. divided the patients in their cohort into three groups. The median survival rate was 273 days for patients who had successful chemotherapy after undergoing PTBD, 65 days for patients who had undergone successful percutaneous transhepatic biliary drainage without receiving subsequent chemotherapy and 34 days in the remaining patients who had undergone unsuccessful percutaneous transhepatic. ${ }^{[23]}$ Van Laethem et al. evaluated 16 patients and found that patients who had additional chemotherapy after complete resolution of jaundice had a longer survival period. ${ }^{[24]}$ In a study done by Teixeira and colleagues, the median survival period after PTBD was 2.9 months, more than half of their cohort received subsequent chemotherapy. ${ }^{[11]}$ In another study, Zhang et al. patients who received additional treatments (chemotherapy, palliative surgery and other local types) exhibited a significantly longer survival time of 285 days (95\% CI, 218352 days) compared with 150 days (95\% CI, 123177 days) for those without subsequent treatment. ${ }^{[13]}$ However, in a retrospective study with 18 patients by Iwasaki et al., the median survival rate after PTBD was 59, with only two patients receiving chemotherapy after the procedure. ${ }^{[25]}$

In our study, the median total bilirubin level before the intervention was $15.01 \mathrm{mg} / \mathrm{dL}$, which dropped to $6.4 \mathrm{mg} / \mathrm{dL}$ after the intervention $(P<0.01)$. Though some reports suggested that patients with advanced malignant biliary obstruction, who were beyond chemotherapy, should not be offered PTBD; $;^{[1]]}$ other studies have shown improvement in pruritus and other high-bilirubin symptoms post procedure due to drop in bilirubin levels. ${ }^{[14,26]}$ In one study, the patients' quality of life was observed to improve after PTBD despite that serum bilirubin level did not drop down to normal; where mean serum bilirubin level before PTBD was $19.85 \mathrm{mg} / \mathrm{dL}$ and after the procedure at 1 month it became $6.02 \mathrm{mg} / \mathrm{dL} .{ }^{[27]}$ Unfortunately, due to the retrospective nature of our study, we could not evaluate for patients' quality of life, pre-and post-procedure.

The presence of ascites was significantly associated with shorter survival. Our results showed increased serum INR to be significantly associated with longer survival, this is a clinically non-relevant result and we believe it is an artifact. In a study done by Castoldi et al., 93 patients with obstructive jaundice (86 cases due to neoplasm) were treated with PTBD; the predictive factors of short survival time were found to be: serum indirect bilirubin, serum cholinesterase, white blood cell counts, blood urea nitrogen (BUN) levels, histotype of metastasis, and large neoplastic volume. ${ }^{[19]}$ Another report found that palliative chemotherapy after procedure and no hospital readmissions were related to better survival outcome. ${ }^{[1]}$ Kasugo et al. found 6 poor prognostic factors in PTBD; poor performance status, multiple liver metastases, presence of ascites, multiple prior chemotherapy administrations, undifferentiated type histology and high serum CA19-9 level were independently associated with a poor prognosis. In their report, they further developed a prognostic index calculated based on the number of these six factors and was used to classify the patients into a good-risk group (index $\leq 2$ ) and a poor-risk group (index $\geq 3$ ). The median survival time and 2-month survival rate for the two groups were 163 and 44 days, respectively, and 85.7 and 33.3\%, respectively $(P=0.001) \cdot{ }^{[23]}$

One of the strengths of this study is that all PTBD were done by the same interventional radiologists, and hence, there is no role for operator difference in the outcomes measured. Second, there was no missing pre-and postprocedure data. We need to acknowledge the limitations of this study. First, the retrospective nature of the study that hindered measuring the effect of other potential variables and outcomes. Second, the small sample size; this study involved a cohort over a ten-years' time period; this calls for the need for a prospective multicenter study.

\section{Conflict of interests}

The authors declare no conflicts of interest

\section{ACKNOWLEDGEMENTS}

Author would like to acknowledge the help from Mrs. Fatima Abu Touq from Medical records department at King Hussein Cancer Center, Amman-Jordan. 


\section{REFERENCES}

1. Nurick AW, Patey DH, Whiteside CG. Percutaneous transhepatic cholangiography in the diagnosis of obstructive jaundice. Br J Surg 1953; 41: 27-30.

2. Shaldon S, Barber KM, Young WB. Percutaneous transhepatic cholangiography. A modified technique. Gastroenterology 1962; 42: 371-9.

3. Zinberg SS, Berk JE, Plasencia H. Percutaneous transhepatic cholangiography: its use and limitations. Am J Dig Dis 1965; 10: 154-69.

4. Zhong SX. Percutaneous transhepatic cholangiography (PTC) and bile drainage (PTCD): a report of 70 cases (author's transl). Zhonghua wWai Ke Za Zhi 1981; 19: 387-90.

5. Shimaguchi S, Ariyama J. Value of percutaneous transhepatic cholangiography with drainage and ultrasonography in the treatment of extrahepatic bile duct obstruction. J Belge Radiol 1982; 65: 221-5.

6. Chen XR, Ni XY, Huang RX, Wang ZY, Gu J, Lin G, et al. Percutaneous transhepatic cholangiography and drainage. Zhonghua fang she xue za zhi 1983; 17: 33-7.

7. Chandrashekhara SH, Gamanagatti S, Singh A, Bhatnagar S. Current status of percutaneous transhepatic biliary drainage in palliation of malignant obstructive jaundice: A review. Indian J Palliat Care 2016;22:378.

8. Garcarek J, Kurcz J, Guzinski M, Janczak D, Sasiadek M. Ten years single center experience in percutaneous transhepatic decompression of biliary tree in patients with malignant obstructive jaundice. Adv Clin Exp Med 2012; 21: 621-32.

9. van Delden OM, Lameris JS. Percutaneous drainage and stenting for palliation of malignant bile duct obstruction. Eur Radiol 2008; 18: 448-56.

10. Berberat PO, Künzli BM, Gulbinas A, Ramanauskas T, Kleeff J, Müller MW, et al. An audit of outcomes of a series of periampullary carcinomas. Eur J Surg Oncol 2009; 35: 187-91.

11. Teixeira MC, Mak MP, Marques DF, Capareli F, Carnevale FC, Moreira AM, et al. Percutaneous transhepatic biliary drainage in patients with advanced solid malignancies: prognostic factors and clinical outcomes. J Gastrointest Cancer 2013; 44: 398-403.

12. Leng JJ, Zhang N, Dong JH. Percutaneous transhepatic and endoscopic biliary drainage for malignant biliary tract obstruction: a meta-analysis. World J Surg Oncol 2014; 12: 272.

13. Zhang GY, Li WT, Peng WJ, Li GD, He XH, Xu LC. Clinical outcomes and prediction of survival following percutaneous biliary drainage for malignant obstructive jaundice. Oncol Lett 2014; 7: 1185-90.

14. Robson PC, Heffernan N, Gonen M, Thornton R, Brody LA, Holmes R, et al. Prospective study of outcomes after percutaneous biliary drainage for malignant biliary obstruction. Ann Surg Oncol 2010; 17: 2303-11.

15. Dy SM, Harman SM, Braun UK, Howie LJ, Harris PF, Jayes RL. To stent or not to stent: an evidence-based approach to palliative procedures at the end of life. J Pain Symptom Manage 2012; 43: 795-801.
16. Tsetis D, Krokidis M, Negru D, Prassopoulos P. Malignant biliary obstruction: the current role of interventional radiology. Ann Gastroenterol 2016; 29: 33-6.

17. Saluja SS, Gulati M, Garg PK, Pal H, Pal S, Sahni P, et al. Endoscopic or percutaneous biliary drainage for gallbladder cancer: a randomized trial and quality of life assessment. Clin Gastroenterol Hepatol 2008; 6: 944-50.e3.

18. Luman W, Cull A, Palmer KR. Quality of life in patients stented for malignant biliary obstructions. Eur J Gastroenterol Hepatol 1997; 9: 481-4.

19. Castoldi MC, Pisani P, Ideo G, Bellomi M, Cozzi G, Severini A. Prognostic factors after percutaneous transhepatic biliary drainage. Radiol Med 1991; 81: 695-8.

20. Gwon DI, Ko GY, Sung KB, Yoon HK, Kim KA, Kim YJ, et al. Clinical outcomes after percutaneous biliary interventions in patients with malignant biliary obstruction caused by metastatic gastric cancer. Acta Radiol, 2012 53: 422-9.

21. Hatzaras I, George N, Muscarella P, Melvin WS, Ellison EC, Bloomston M. Predictors of survival in periampullary cancers following pancreaticoduodenectomy. Ann Surg Oncol 2010; 17: 991-7.

22. Thornton RH, Ulrich R, Hsu M, Moskowitz C, Reidy-Lagunes D, Covey $\mathrm{AM}$, et al. Outcomes of patients undergoing percutaneous biliary drainage to reduce bilirubin for administration of chemotherapy. J Vasc Interv Radiol 2012; 23: 89-95.

23. Kasuga A, Ishii H, Ozaka M, Matsusaka S, Chin K, Mizunuma N, et al. Clinical outcome of biliary drainage for obstructive jaundice caused by colorectal and gastric cancers. Jpn J Clin Oncol 2012; 42: 1161-7.

24. Van Laethem JL, De Broux S, Eisendrath P, Cremer M, Le Moine O, Deviere J. Clinical impact of biliary drainage and jaundice resolution in patients with obstructive metastases at the hilum. Am J Gastroenterol 2003; 98: 1271-7.

25. Iwasaki M, Furuse J, Yoshino M, Konishi M, Kawano N, Kinoshita T, et al. Percutaneous transhepatic biliary drainage for the treatment of obstructive jaundice caused by metastases from nonbiliary and nonpancreatic cancers. Jpn J Clin Oncol 1996; 26: 465-8.

26. Larssen L, Medhus AW, Hjermstad MJ, Körner H, Glomsaker T, Søberg T, et al. Patient-reported outcomes in palliative gastrointestinal stenting: a Norwegian multicenter study. Surg Endosc 2011; 25: 3162-9.

27. Gamanagatti S, Singh T, Sharma R, Srivastava DN, Dash NR, Garg PK. Unilobar Versus Bilobar Biliary Drainage: Effect on Quality of Life and Bilirubin Level Reduction. Indian J Palliat Care 2016; 22: 50-62.

How to cite this article: Tuqan W, Innabi A, Alawneh A, Farsakh FA, AlKhatib M. Prediction of survival following percutaneous biliary drainage for malignant biliary obstruction. J Transl Intern Med 2017; 5: 127-131. 